\title{
Prognostic significance and role in TNM stage of tumor deposits in esophageal cancer
}

\author{
Qi-Xin Shang ${ }^{1 *}$, Yu-Shang Yang ${ }^{1 *}$, Li-Yan $\mathrm{Xu}^{2,3}$, En-Min $\mathrm{Li}^{2,4}$, Wei-Peng Hu${ }^{1}$, Long-Qi Chen ${ }^{1}$ \\ ${ }^{1}$ Department of Thoracic Surgery, West China Hospital of Sichuan University, Chengdu 610041, China; ${ }^{2}$ The Key Laboratory of Molecular Biology \\ for High Cancer Incidence Coastal Chaoshan Area, ${ }^{3}$ Institute of Oncologic Pathology, ${ }^{4}$ Department of Biochemistry and Molecular Biology, Shantou \\ University Medical College, Shantou 515041, China \\ Contributions: (I) Conception and design: LQ Chen, QX Shang, YS Yang; (II) Administrative support: WP Hu; (III) Provision of study materials or \\ patients: LY Xu, EM Li; (IV) Collection and assembly of data: YS Yang, EM Li; (V) Data analysis and interpretation: QX Shang, LQ Chen; (VI) \\ Manuscript writing: All authors; (VII) Final approval of manuscript: All authors. \\ *These authors contributed equally to this work. \\ Correspondence to: Prof. Long-Qi Chen, MD, PhD. Department of Thoracic Surgery, West China Hospital of Sichuan University, No. 37, Guoxue \\ Alley, Chengdu 610041, China. Email: drchenlq@scu.edu.cn.
}

\begin{abstract}
Background: Tumor deposits (TDs) are now observed in esophageal cancer (EC), but the role of TDs is seldom elucidated. This study aimed to research the prognostic significance and the role of TDs in EC. The patients with primary EC, who had undergone curative esophagectomy in West China Hospital from May 2005 to May 2011 were retrospectively enrolled.

Methods: The prognosis and clinicopathological traits were compared between tumor deposits positive (TDP) and tumor deposits negative (TDN) groups in all patients and TNM 0-IV stages respectively.

Results: In our study, 1,044 patients were enrolled, with 948 (90.8\%) in TDN group and 96 (9.2\%) in TDP group. TDP group had significantly more advanced EC and worse prognosis (all $\mathrm{P}<0.001)$ than TDN group in all patients, TNM II stage and TNM III stage. The prognosis of TDP group in TNM II stage was significantly worse than TDN patients in TNM III stage $(\mathrm{P}<0.001)$, and the worst prognosis was always found in patients with at least one TD regardless of the number of metastatic lymph node is.

Conclusions: Patients in TDP subgroup had more advanced EC and worse prognosis than those in TDN subgroup. It might be more reasonable to be regarded as an indicator of stage migration in EC.
\end{abstract}

Keywords: Tumor deposits (TDs); esophageal carcinoma; prognosis; TNM stage

Submitted Jun 27, 2017. Accepted for publication Oct 10, 2017.

doi: $10.21037 /$ jtd.2017.10.60

View this article at: http://dx.doi.org/10.21037/jtd.2017.10.60

\section{Introduction}

It's believed that esophageal cancer (EC) is one of the most malignant tumors in the world $(1,2)$. Among all of the clinical and pathological characters of the EC, lymph node metastasis is regarded as one of the strongest prognostic factors in patients with EC (3) and such as the number, extent even the size of the metastatic lymph node and extranodal vascular tumor embolus have been reported to be related to poor prognosis in EC (3-5). However, the tumor deposits (TDs), which are usually found in colorectal cancer, are newly observed in EC, and there are no published series reported the prognostic role in EC. Some scholars usually regarded esophageal TDs as extranodal invasion (6), which is usually defined as the tumor cells invade through the lymph node capsules into the perinodal tissue and it includes the invasive tissue connected with the original lymph nodes which is so called the lymph node capsular invasion (7). However, the TDs was defined as isolated tumor foci found in the peri-organic fat or perinodal fat away from the leading edge of the tumor and with no evidence of residual lymph node tissue according to the 
American Joint Committee on Cancer Staging Manual, 8th edition (AJCC 8TNM) (8) and College of American Pathologists cancer protocol (CAPCC) (9). There are several series reported the extranodal invasion is associated with the poor prognosis in patients with EC $(7,10-12)$, however they didn't take the TDs apart from the extranodal invasion. At the same time, neither are available researches reported the relationship between the extranodal invasion and the TNM stage in EC even in colorectal carcinoma nor the association between the TDs and the TNM stage in EC. Therefore, we retrospectively investigated 1,044 selected patients with EC surgically resected and evaluated the role TDs play in TNM stage of EC as well as the prognostic significance of TDs in patients with EC.

\section{Methods}

\section{Patients}

A total of 1,044 consecutive patients with EC who had underwent the curative esophagectomy from May 2005 to May 2011 at the Department of Thoracic Surgery, West China Hospital of Sichuan University were investigated. The research was approved by the human participants committee of West China Hospital of Sichuan University (the ethical number: 2005-126), and all patients were informed the risk of the operation. The use of their resected specimens, and the written consents were obtained preoperatively. At the same time, all the surgical procedures patients had received, tumor staging and specimens' examination were referred to the American Joint Committee on Cancer Staging Manual, 8th edition (AJCC 8th TNM) and Union for International Cancer Control protocol (UICC). Meanwhile, the informed consent was obtained from all subjects preoperatively. The exclusion criteria are: (I) patients who were lost to follow-up; (II) palliative surgery and R1 or R2 resection; (III) patients who had accepted the preoperative neoadjuvant chemotherapy or radiotherapy. And finally, there are 948 patients (90.8\%) enrolled in TDN group and 96 patients $(9.2 \%)$ distributed to TDP group. The middle age of these 1,044 patients is 60 years old with the range from 20 to 90 years old and the median follow-up time is 50.40 months with the rage from 2.00 to 117.43 months.

\section{Surgical procedures and bistologic examination}

Among the included 1,044 patients, the surgical procedures selection depended on patients' images of computed tomography, magnetic resonance imaging, specific $\mathrm{X}$-ray and cervical ultrasonography, at the same time, surgeons in our hospital would evaluate the patients' general condition and finally supplied for the patients the most appropriate surgical procedures. In 921 suitable patients (88.2\%), Sweet esophagectomy was performed and twofield lymph node dissection (thoracoabdominal) was also conducted. Meanwhile, 101 patients (9.7\%) had accepted the Ivor-Lewis esophagectomy and the two-field lymph node dissection was performed as well. In addition, nine patients $(8.6 \%)$ diagnosed as the pure esophagogastric junction carcinoma preoperatively were conducted the trans-abdominal esophagectomy and two patients $(0.2 \%)$ had received the transhiatal esophagectomy. The dissected lymph nodes were separated from the resected esophagus and peri-esophagus tissues, which were marked and indicated the location according to the guideline of AJCC 8th TNM and UICC protocol. The mean number of dissected lymph nodes is 15 per patient [0-62]. Two experienced pathologists would fix the resected specimens, embedded and stained them with diaminobenzidine chromogen counterstained solution [1:50, EnVisionTM Detection Kit, Gene Tech (Shanghai) Company Limited] and hematoxylin (Zhongshan Golden Bridge Biotechnology Co., Ltd, Beijing, China) subsequently. The routine way of assessing each specimen was adopted histologically, and the pathologists would document the extent and location of metastatic lymph nodes by examining the largest cross section of dissected lymph nodes.

\section{Definition of TDs}

Although there is no specific definition of TDs in EC, we drew lessons from the definition in the AJCC 8th TNM and CAPCC protocol, which defined the TDs as isolated tumor foci found in the peri-organic fat or peri-nodal fat away from the leading edge of the tumor and with no evidence of residual lymph node tissue. And the results considered as the extranodal invasion or the lymph node capsular invasion was not regarded as the TDs. About 65 patients with TDs were obtained from reexamining the diaminobenzidine and hematoxylin stained specimens of 468 patients with positive lymph node metastasis, and there are 31 patients divided into TDP group from reexamined the diaminobenzidine and hematoxylin stained specimens of 576 patients with negative lymph node metastasis as well. The pathological histology of TD in two examples was shown in Figure 1, in which the absence of a capsule or recognizable lymph nodal 

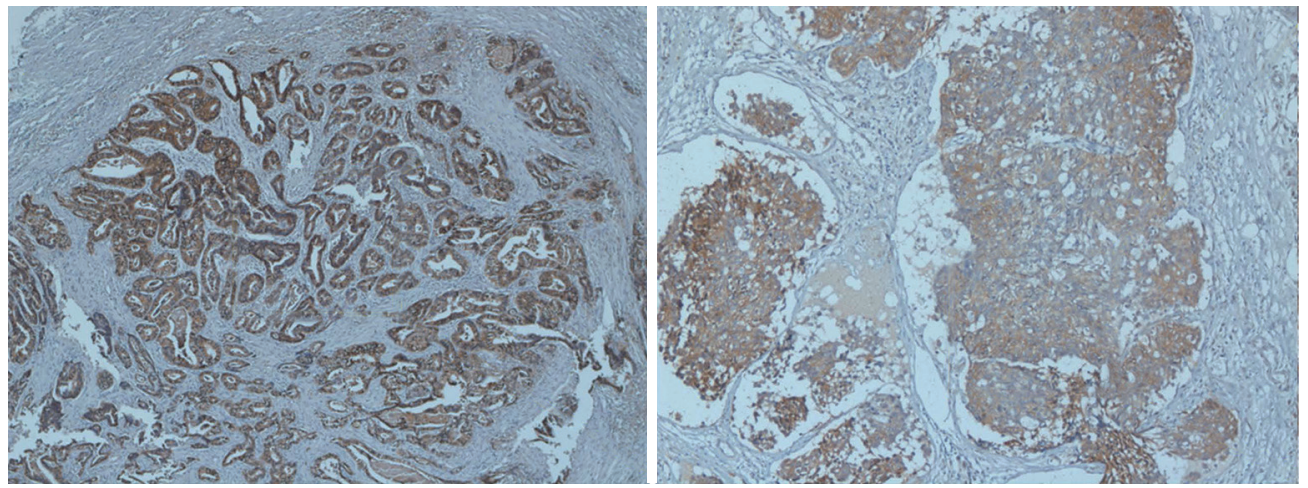

Figure 1 The pathological histology of TD in two examples. Note the absence of a capsule or recognizable lymph nodal architecture. The specimens were embedded and stained by diaminobenzidine chromogen counterstained solution [1:50, EnVisionTM Detection Kit, Gene Tech (Shanghai) Company Limited] and hematoxylin (Zhongshan Golden Bridge Biotechnology Co., Ltd., Beijing, China) subsequently. TD, tumor deposit.

architecture can be noted.

\section{Statistical analysis}

The clinicopathologic features between the TDP and TDN group and among different TNM stage groups were analyzed by means of Fisher exact test and Chi-square test. The X-tail software (Version 3.6.1) was used to calculate the optimal cut-point of the number of TDP. The overall survival and 1-, 2-, 3-, 4- and 5-year survival were exhibited from the KaplanMeier curves and the log-rank test was used to determine the statistical significance. Multivariate survival analysis was figured out through the Cox proportional hazard regression model. At the same time, in order to analysis the relationship between lymph node metastasis and TDs, the method of 1:2 propensity score matching was used. The statistical significance was regarded as the $\mathrm{P}$ value $<0.05$. All methods were performed in accordance with the relevant guidelines and regulations and the statistical analysis were conduct by $\mathrm{IBM}^{\circledR}$ SPSS $^{\circledast}$ Statistics Version 21.0.

\section{Results}

\section{All patients}

There are 1,044 patients with EC and TDs were found in 96 patients $(9.19 \%)$. In order to analyze the significance of clinicopathologic features and the survival outcomes between patients with or without TDs, we divided all patients into two groups: tumor deposits positive (TDP) and tumor deposits negative (TDN) group. The clinicopathologic features between two groups were shown in Table 1. There are significantly more male patients $(\mathrm{P}=0.018)$, more patients with peripheral vascular diseases $(\mathrm{P}=0.002)$, advanced $\mathrm{T}$ stage $(\mathrm{P}=0.009)$, advanced $\mathrm{N}$ stage $(\mathrm{P}<0.001)$ and $\mathrm{TNM}$ stage $(\mathrm{P}<0.001)$ in TDP group than in TDN group, in which only TNM stage was analyzed as an independent factor $[\mathrm{P}=0.016$; odds ratio $(\mathrm{OR})=2.557$, 95\% CI: 1.191-5.487]. The median follow-up time in TDP and TDN group are 40.47 and 64.23 months respectively. And the median survival time is 11.40 months in TDP group and 36.30 months in TDN group. A big decreasing of survival rates can be seen in Table 2, in which the 1- to 5 -year survival rates are from $47.9 \%$ to $0 \%$ in TDP group respectively compared with $83.4 \%$ to $39.1 \%$ in TDN group. The Kaplan-Meier curves showed that patients in TDP group will get significant worse prognosis than patients in TDN group $(\mathrm{P}<0.001$ in Figure $2 A)$. Meanwhile the Cox proportional hazard regression indicated that TDs $[\mathrm{P}<0.001$; hazard ratio $(\mathrm{HR})=2.633,95 \% \mathrm{CI}: 1.386-5.001]$ was an independent prognostic factor in patients with $\mathrm{EC}$ as well as the age ( $\mathrm{P}=0.039$; HR $=1.185,95 \%$ CI: 1.008-1.393), T stage $(\mathrm{P}=0.028 ; \mathrm{HR}=1.166,95 \% \mathrm{CI}: 1.016-1.337), \mathrm{N}$ stage $(\mathrm{P}=0.035$; HR $=1.148,95 \% \mathrm{CI}: 1.010-1.304)$ and $\mathrm{M}$ stage $(\mathrm{P}=0.014 ; \mathrm{HR}=2.011,95 \% \mathrm{CI}: 1.149-3.520)$ (Table 3).

\section{TNM 0-II stages}

Among 1,044 patients with EC, there are $9(0.86 \%)$ patients in TNM stage 0 and $84(8.04 \%)$ patients in TNM stage I pathologically, however, there is no TDs found in these 93 patients. Nevertheless, there are 449 patients in TNM stage II, among them, 26 patients $(5.79 \%)$ found TDs 


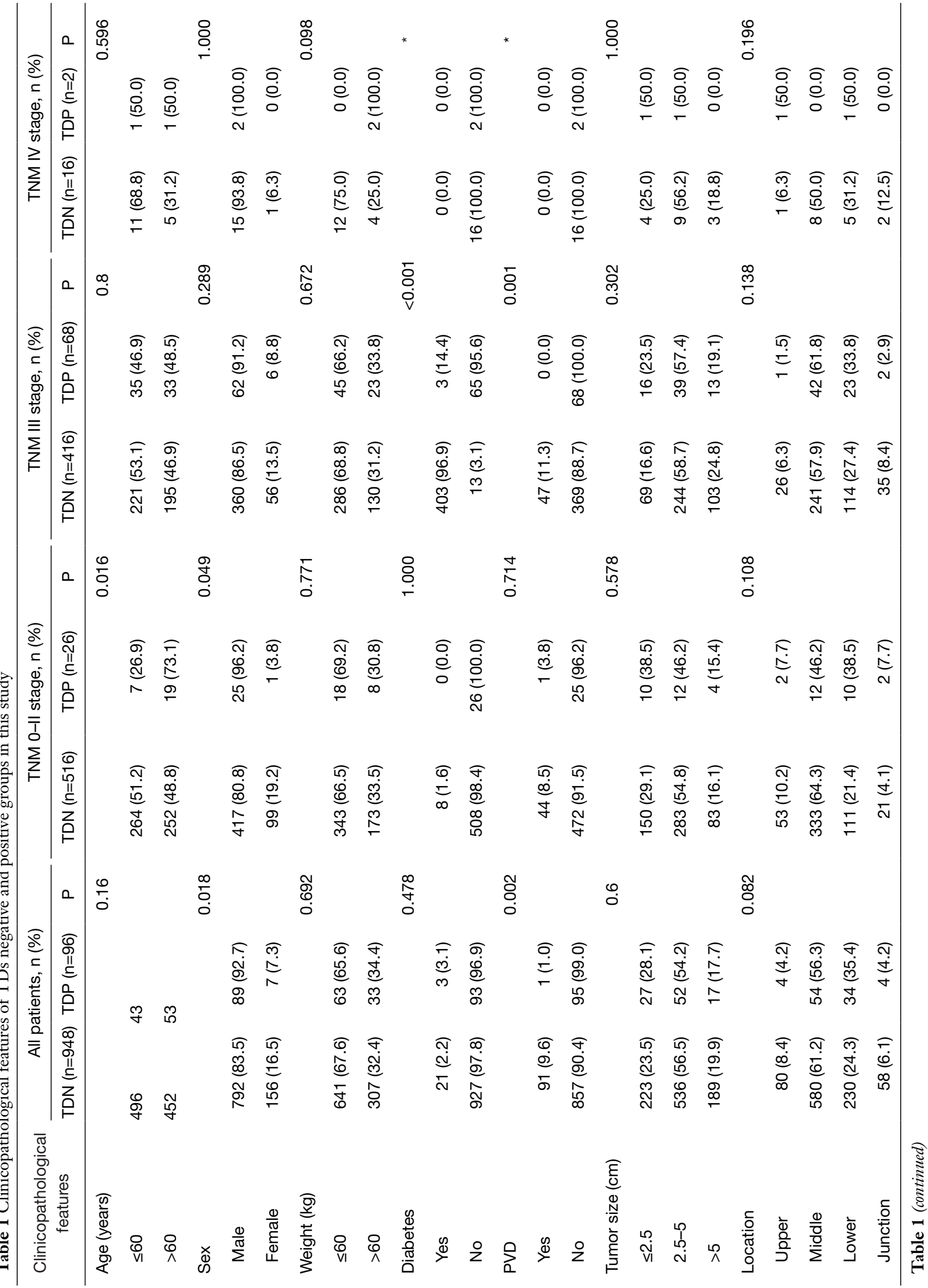




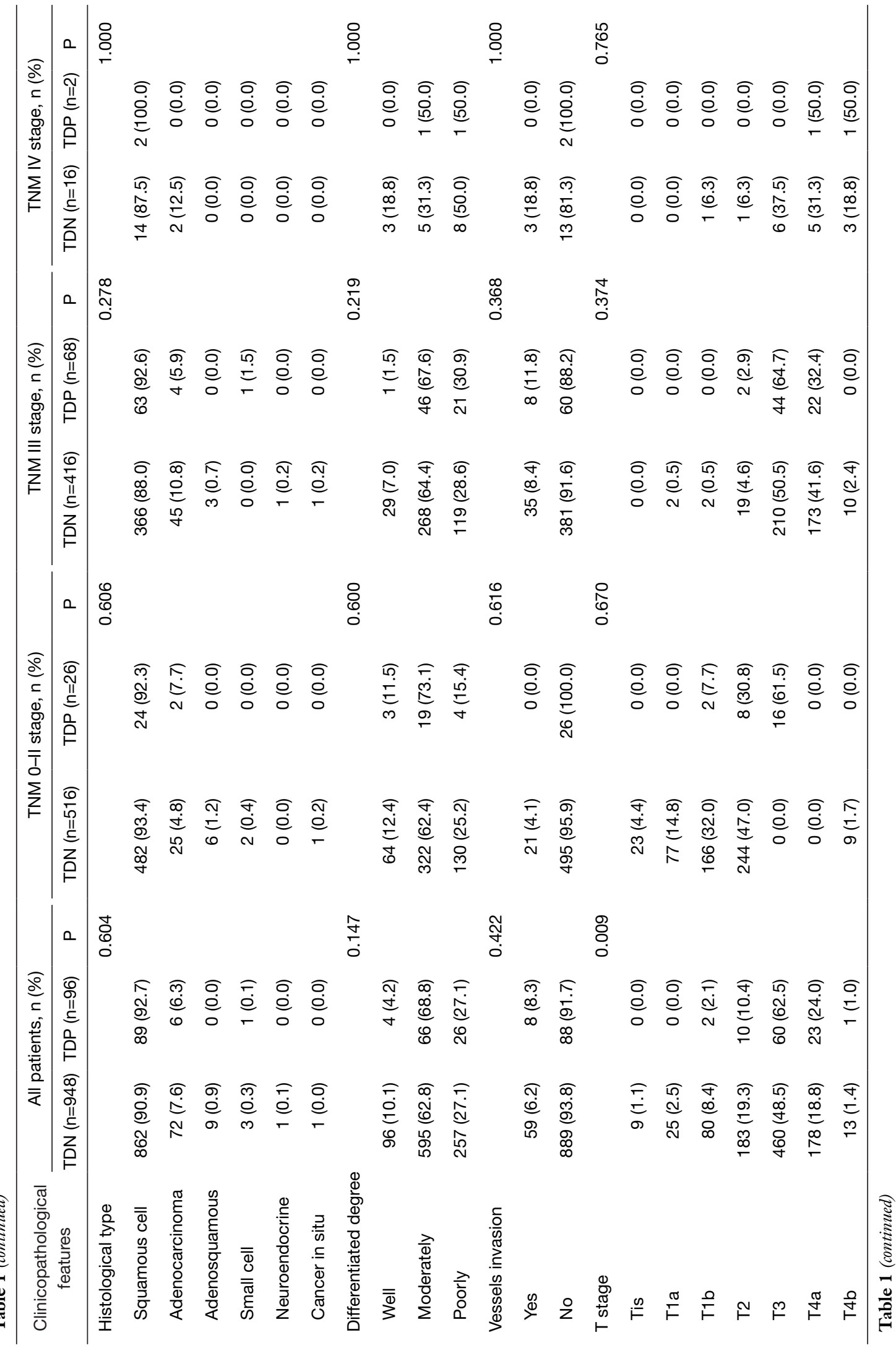




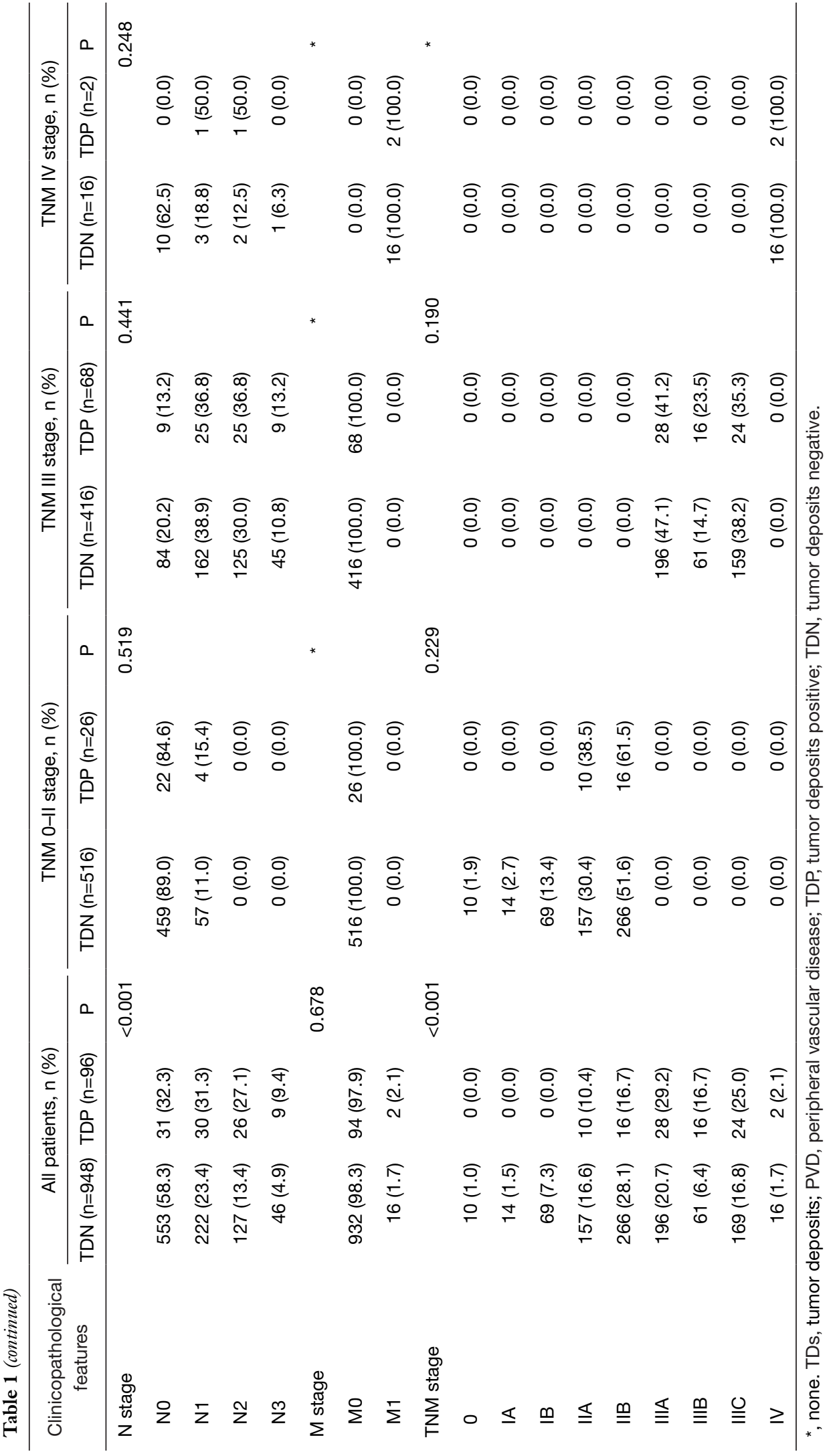




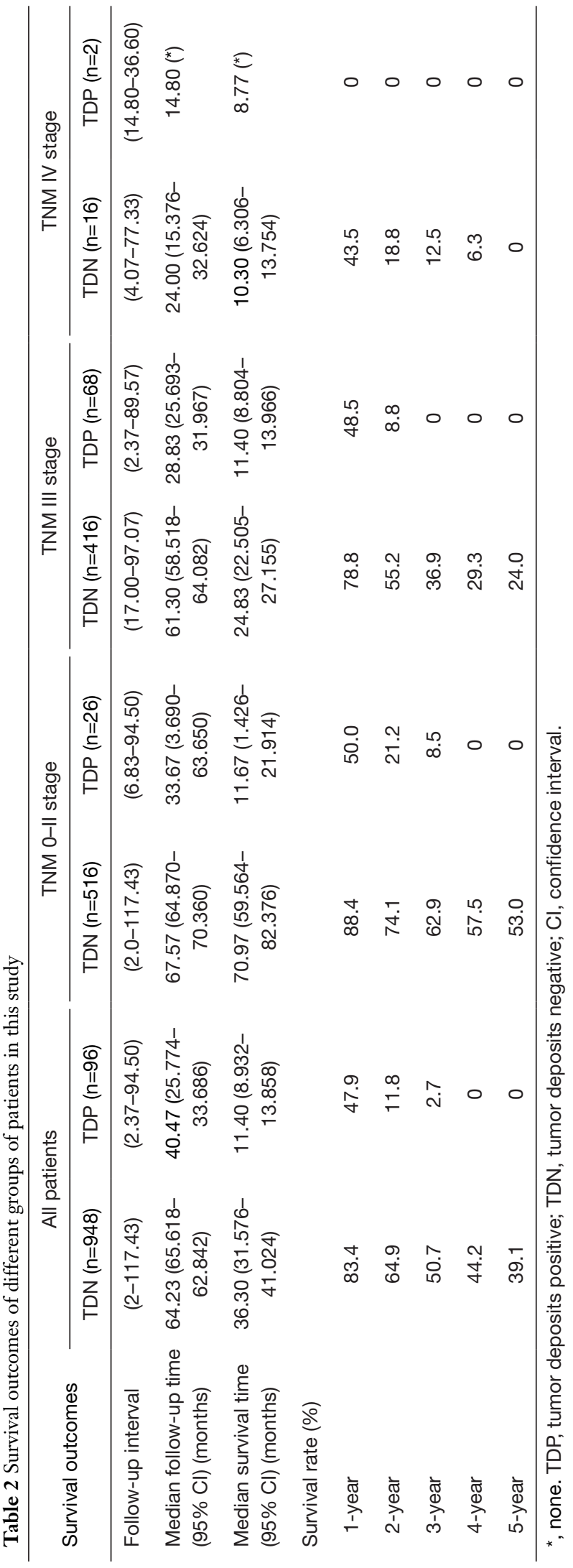

were divided into TDP group and the last 423 patients in TNM stage II without TDs were combined with patients in TNM stage 0 and TNM stage I. It can be elucidated that only more patients in old age $(\mathrm{P}=0.016)$ and more male patients $(\mathrm{P}=0.049)$ were found in TDP group than in TDN group significantly, and there're no differences in other clinicopathologic features (Table 1). Logistic regression showed that only age was an independent factor related to TDP group ( $\mathrm{P}=0.018$; OR $=3.224$, 95\% CI: $1.227-8.473)$. In TDP group, the median follow-up time and the median survival time are 33.67 and 11.67 months compared with 67.57 and 70.97 months in TDN group respectively. The 1 - to 5 -year survival rates are from $50 \%$ to $0 \%$ in TDP group respectively and from $88.4 \%$ to $53 \%$ in TDN group by contrast (Table 2). At the same time the Kaplan-Meier survival curves showed that the prognosis of TDP group in TNM stage $0-$ II was significantly worse than TDN group in same stage $(\mathrm{P}<0.001$ in Figure $2 A)$. Further we found age $(\mathrm{P}=0.028 ; \mathrm{HR}=1.346,95 \% \mathrm{CI}: 1.032-1.757)$ was an independent prognostic factor, as well as $\mathrm{T}$ stage $(\mathrm{P}<0.001$; $\mathrm{HR}=1.562,95 \%$ CI: $1.257-1.942), \mathrm{N}$ stage $(\mathrm{P}=0.001$; $\mathrm{HR}=1.563$, 95\% CI: $1.198-2.093)$ and differentiation grade $(\mathrm{P}=0.049 ; \mathrm{HR}=1.244,95 \% \mathrm{CI}: 1.001-1.545)$ (Table 3).

\section{TNM III stage}

There are 484 patients with EC in TNM stage III, in which 68 (14.05\%) patients were divided into TDP group. There are more patients with diabetes $(\mathrm{P}<0.001)$ and more patients with peripheral vascular disease $(\mathrm{P}=0.001)$ in TDP group than in TDN group significantly (Table 1). The median follow-up time is 28.83 months in TDP group and 61.30 months in TDN group respectively. The median survival time in TDP group and TDN group are 11.40 and 24.83 months. The 1 - to 5 -year survival rates are from $48.5 \%$ to $0 \%$ in TDP group when compared with $78.8 \%$ to $24.0 \%$ in TDN group (Table 2). It can be concluded that TDP patients had gotten worse prognosis than patients in TDN group $(\mathrm{P}<0.001$ in Figure $2 C)$. And only differentiation grade was identified as the independent prognostic factor $(\mathrm{P}=0.042$; HR $=2.228,95 \% \mathrm{CI}$ : 1.029-4.826) (Table 3).

\section{TNM IV stage}

When take TNM stage IV for consideration, there are 18 patients with EC meet the inclusion criteria, in which, only 2 patients had been found the TDs postoperatively. 

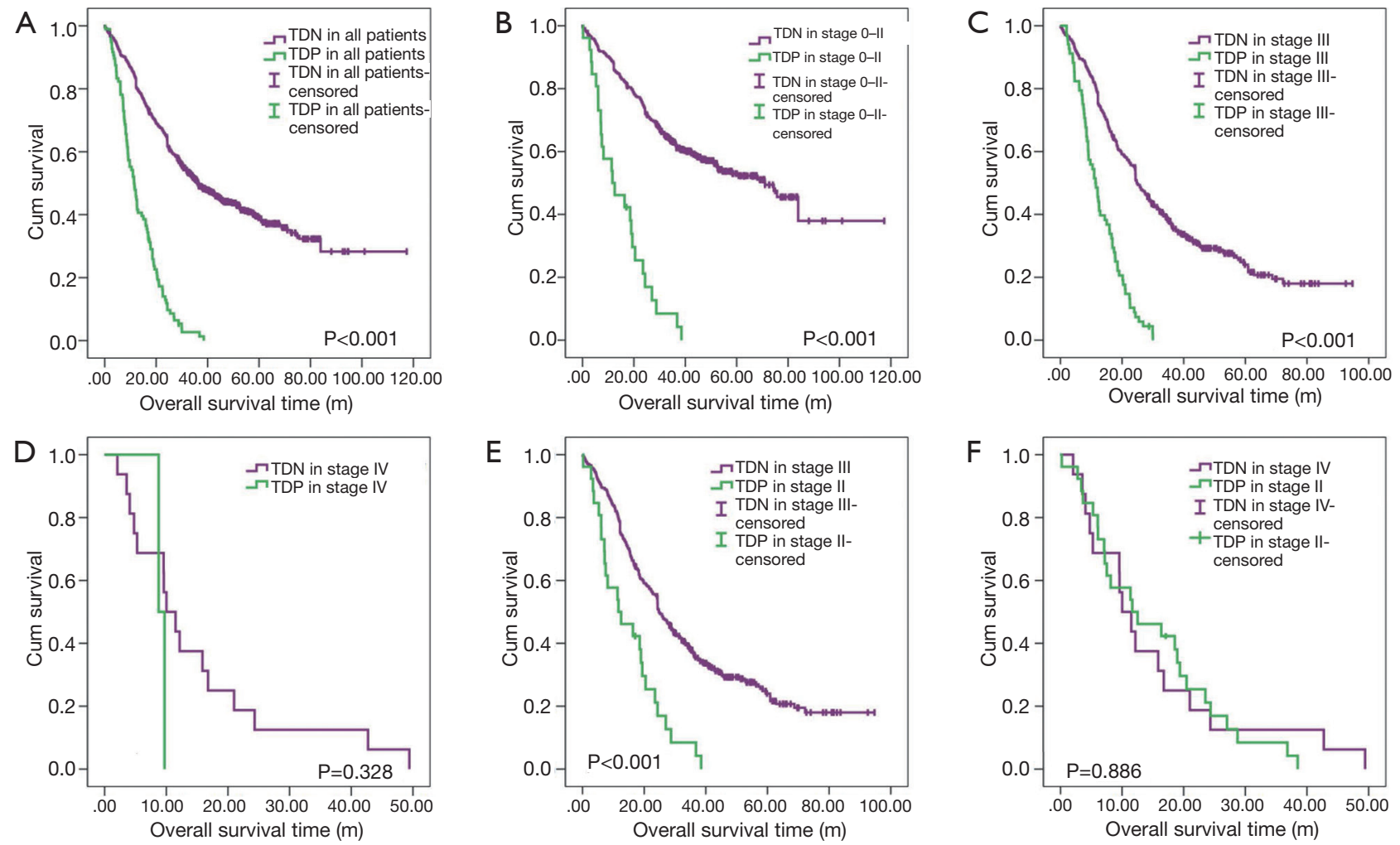

Figure 2 Prognosis of TDN and TDP groups in all patients, TNM 0-II, III and IV stages, and comparison on prognosis of TDP in TNM II with TDN patients in TNM III and IV patients. (A) In all patients, TDP patients got significant worse prognosis than TDN patients $(\mathrm{P}<0.001)$; (B) in TNM 0-II stage, TDP patients got worse prognosis than patients in TDN group $(\mathrm{P}<0.001)$; (C) in TNM III stage, TDP patients got worse prognosis than patients in TDN group $(\mathrm{P}<0.001)$; (D) in TNM IV stage, no significant difference on prognosis between TDP and TDN groups was found ( $\mathrm{P}=0.328)$; (E) TDP patients in TNM stage II had obviously worse survival outcome than patients of TDN in TNM stage III $(\mathrm{P}<0.001)$; $(\mathrm{F})$ no significant prognosis was found between the TDP patients in TNM stage II and TDN patients in TNM IV $(\mathrm{P}=0.886)$. TDP, tumor deposits positive; TDN, tumor deposits negative.

However, there is no significant difference between TDP and TDN group in clinicopathologic features listed in Table 1. The median follow-up time is 14.80 months in TDP group and 24.00 months in TDN group. The median survival time is 8.77 months in TDP group and 10.30 months in TDN group. The 1 - to 5 -year survival rates in TDP group and in TDN group are total $0 \%$ and from $43.5 \%$ to $0 \%$ respectively (Table 2). From the Kaplan-Meier survival curves we concluded that there is no significant difference on prognosis between TDP and TDN groups $(\mathrm{P}=0.328$ in Figure 2D), moreover, no independent prognostic factor was found from the results of Cox proportional hazard regression neither (Table 3).

\section{The relationship between the TDP and TNM stage}

As it was mentioned before that there is significant difference of prognosis between TDP patients and TDN patients. At the same time, the cross-stage comparison was also performed in order to clarify if TDs of EC influence the tumor stage or not, and it can be illustrated from Kaplan-Meier survival curves that TDP patients in TNM stage II had obviously worse survival outcome than patients of TDN in TNM stage III $(\mathrm{P}<0.001$ in Figure $2 E)$, nevertheless, no significant prognosis was found between the TDP patients in TNM stage II and TDN patients in TNM IV $(\mathrm{P}=0.886$ in Figure $2 F)$.

\section{The relationship between the TDP and lymph node metastasis}

Now that the TDs and $\mathrm{N}$ stage were confirmed as the independent prognostic factor in patients with EC, the 


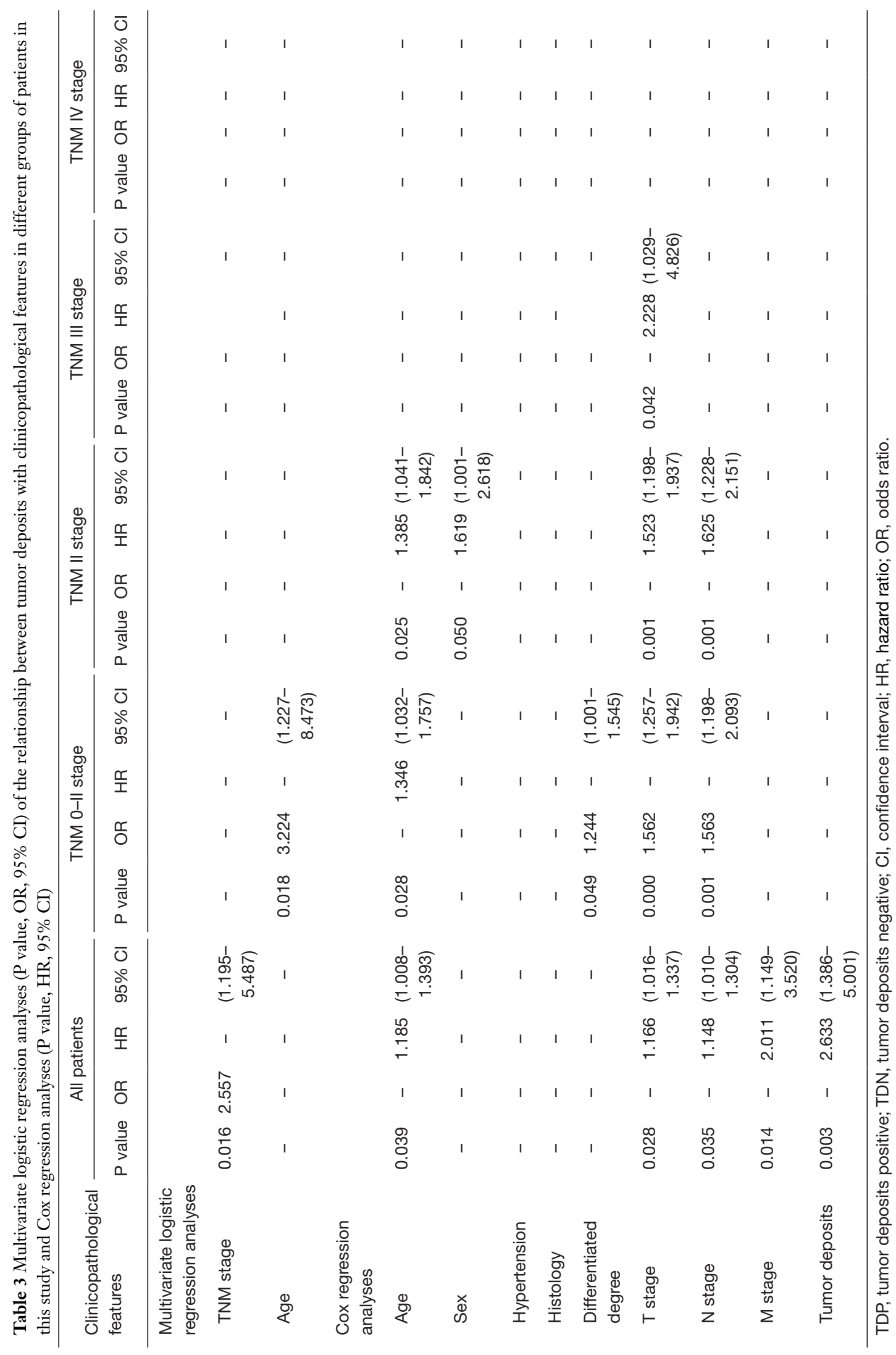



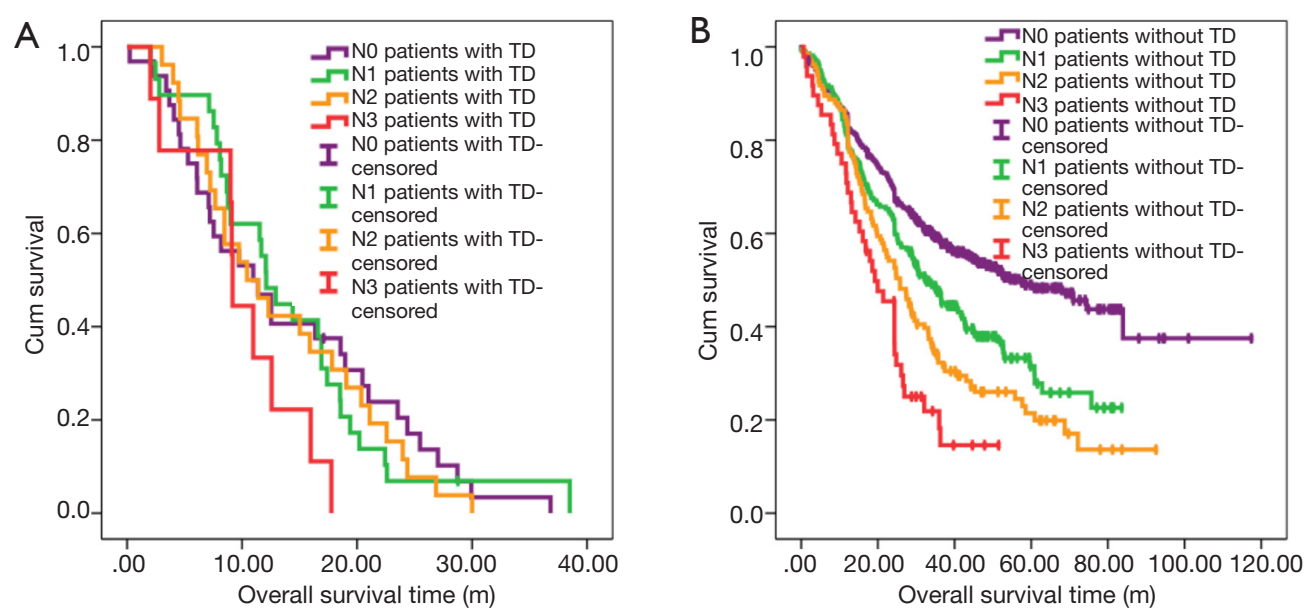

\begin{tabular}{lc}
\hline & P value \\
\hline$P$ (N0 vs. N1) & 0.964 \\
$P$ (N0 vs. N2) & 0.654 \\
$P$ (N0 vs. N3) & 0.139 \\
$P$ (N1 vs. N2) & 0.892 \\
$P$ (N1 vs. N3) & 0.109 \\
$P$ (N2 vs. N3) & 0.158 \\
\hline
\end{tabular}

\begin{tabular}{ll}
\hline & P value \\
\hline$P$ (N0 vs. N1) & 0.000 \\
$P$ (N0 vs. N2) & 0.000 \\
$P$ (N0 vs. N3) & 0.000 \\
P (N1 vs. N2) & 0.031 \\
P (N1 vs. N3) & 0.000 \\
$P$ (N2 vs. N3) & 0.029 \\
\hline
\end{tabular}

Figure 3 Comparison on prognosis of different $\mathrm{N}$ stages between TDP patients and TDN patients. (A) No significant prognostic difference was found from N0 to N3 stage among TDP patients; (B) significant prognostic difference was found from N0 to N3 stage among TDN patients. TDP, tumor deposits positive; TDN, tumor deposits negative.

relationship between both of them is the next point we concerned about. We compared the prognostic difference of $\mathrm{N}$ stage between TDP and TDN patients, and the Kaplan-Meier curves showed that among TDP patients no significant prognostic difference was found from $\mathrm{N} 0$ to $\mathrm{N} 3$ stage (Figure $3 A$ ), however, the completely opposite result was found among TDN patients (Figure 3B). Therefore, we took the method of 1:2 propensity score matching in which one TDP patient would be matched with two TDN patients whose gender, group of age, group of tumor size, tumor location, histological type, differentiated degree, $\mathrm{T}$ stage, $M$ stage, type of postoperative adjuvant therapy, conditions of vessels invasion and tumor recurrence were the same as matched TDP patient. And finally, 192 TDN qualified patients were matched with 96 TDP patients (Table 4). Among these patients, we made a comparison on the number of TDs and the number of metastatic lymph nodes. And the significant difference on prognosis between $\mathrm{TD}=1$, $\mathrm{n}=0$ group and $\mathrm{n}=0$ group $(\mathrm{P}=0.006)$ as well as between $\mathrm{TD}=1, \mathrm{n}=0$ group and $\mathrm{n}=1$ group were found $(\mathrm{P}=0.000)$ (Figure $4 A$ ), in which $\mathrm{TD}=1, \mathrm{n}=0$ represents patients with one tumor deposit but without lymph node metastasis; $\mathrm{n}=0$ represents TDN patients without metastatic lymph node; $\mathrm{n}=1$ represents TDN patients with one metastatic lymph node. No significant difference on prognosis was found between $\mathrm{TD}=1, \mathrm{n}=1$ group and $\mathrm{n}=2$ group $(\mathrm{P}=0.239)$ as well as between $\mathrm{n}=1$ group and $\mathrm{n}=2$ group $(\mathrm{P}=0.153)$ except for groups between $\mathrm{TD}=1, \mathrm{n}=1$ and $\mathrm{n}=2(\mathrm{P}=0.002)$ (Figure $4 B)$. Significant difference on prognosis between $\mathrm{TD}=1, \mathrm{n} \geq 2$ group and $\mathrm{n} \geq 2$ group was found $(\mathrm{P}=0.000)$ (Figure $4 C$ ). Patients in the group of $T D \geq 2, n=0$ got the significantly worst prognosis than patients in $\mathrm{n} \geq 2$ group than patients in $\mathrm{n}=0$ group (all $\mathrm{P}=0.000$ ) (Figure $4 D$ ). Patients in the group of $T D \geq 2, n=1$ got the significantly worst prognosis than patients in $n \geq 2$ group than patients in $n=1$ group (Figure $4 E$ ). Significant difference on prognosis between $T D \geq 2$, $\mathrm{n} \geq 2$ group and $\mathrm{n} \geq 2$ group was found $(\mathrm{P}=0.000)$ (Figure $4 F$ ).

\section{Discussion}

Since Gabriel et al. (13) first proposed the TDs in 1935, the researches of TDs have been continuing all the time. 
Table 4 Baseline clinical characteristics before and after matching

\begin{tabular}{|c|c|c|c|c|c|c|}
\hline Clinical characteristics & \multicolumn{3}{|c|}{ Before matching } & \multicolumn{3}{|c|}{ After matching } \\
\hline Sex & & & 0.018 & & & 0.586 \\
\hline Male & 89 & 792 & & 89 & 178 & \\
\hline Female & 7 & 156 & & 7 & 14 & \\
\hline$\leq 60$ & 43 & 496 & & 43 & 86 & \\
\hline$>60$ & 53 & 452 & & 53 & 106 & \\
\hline Tumor size (cm) & & & 0.600 & & & 0.537 \\
\hline$\leq 2.5$ & 27 & 223 & & 27 & 54 & \\
\hline Surgical procedure & & & 0.492 & & & 0.574 \\
\hline Open & 86 & 842 & & 86 & 172 & \\
\hline Minimal invasive & 10 & 106 & & 10 & 20 & \\
\hline Location & & & 0.082 & & & 0.538 \\
\hline Upper & 4 & 80 & & 4 & 8 & \\
\hline Middle & 54 & 580 & & 54 & 108 & \\
\hline Lower & 34 & 230 & & 34 & 68 & \\
\hline Junction & 4 & 58 & & 4 & 8 & \\
\hline Moderately & 66 & 595 & & 66 & 132 & \\
\hline Poorly & 26 & 257 & & 26 & 52 & \\
\hline Vessels invasion & & & 0.442 & & & 0.597 \\
\hline Yes & 8 & 59 & & 8 & 16 & \\
\hline No & 88 & 889 & & 88 & 176 & \\
\hline
\end{tabular}

Table 4 (countinued) 
Table 4 (countinued)

\begin{tabular}{|c|c|c|c|c|c|c|}
\hline Clinical characteristics & \multicolumn{3}{|c|}{ Before matching } & \multicolumn{3}{|c|}{ After matching } \\
\hline T stage & & & 0.009 & & & 0.538 \\
\hline Tis & 0 & 9 & & 0 & 0 & \\
\hline T1a & 0 & 25 & & 0 & 0 & \\
\hline $\mathrm{T} 2$ & 10 & 183 & & 10 & 20 & \\
\hline T3 & 60 & 460 & & 60 & 120 & \\
\hline $\mathrm{T} 4 \mathrm{a}$ & 23 & 178 & & 23 & 46 & \\
\hline $\mathrm{T} 4 \mathrm{~b}$ & 1 & 13 & & 1 & 2 & \\
\hline M1 & 2 & 16 & & 2 & 4 & \\
\hline $\begin{array}{l}\text { Postoperative adjuvant } \\
\text { therapy }\end{array}$ & & & 0.016 & & & 0.519 \\
\hline No & 53 & 594 & & 53 & 106 & \\
\hline Chemotherapy & 12 & 128 & & 12 & 24 & \\
\hline Radiotherapy & 14 & 57 & & 14 & 28 & \\
\hline Chemoradiotherapy & 17 & 169 & & 17 & 34 & \\
\hline Tumor recurrence & & & 0.014 & & & 0.561 \\
\hline
\end{tabular}

TDP, tumor deposits positive; TDN, tumor deposits negative.

In the study of Gabriel et al. (13) the TDs was regarded as vascular invasion. The consequent results of researches have supported the conclusion of Gabriel, which reported that the most of TDs originated from lymphatic invasion (14-16). Prabhudesai (17) reported the intramural vascular invasion $(\mathrm{P}=0.017)$, extramural vascular invasion $(\mathrm{P}=0.039)$, perineural invasion $(\mathrm{P}=0.039)$, and lymph node involvement $(\mathrm{P}=0.008)$ were associated with the TDs, and all these processes were correlated with poor prognosis $(16,17)$. Puppa (18) had reported that TD were not only limited to colorectal cancer but were also common to different tumor types. Therefore, TDs is not an unfamiliar terminology in the carcinoma of gastrointestinal but is a new word to the world of EC. As many scholars reported that the extranodal invasion was associated with poor prognosis in several advanced EC $(7,10-12)$, however, the extranodal invasion is not only included the cancer cells invade into peri-nodal fatty tissues through the lymph node capsule named lymph node capsular invasion pathologically, but also the individual tissues with the same cancer cells, found adjacent to original EC, defined TDs pathologically were included. Meanwhile the Makoto (7) have already reported the lymph node capsular invasion would bring worse prognosis to the patients with esophageal squamous cell carcinoma and it was an indicator to the distant organ recurrence, therefore the clinicopathologic features and the prognostic effective of TDs are what we concentrated on this time. From our study, we found that patients in TDP group had already been correlated with advanced tumor and poor postoperative prognosis when compared with TDN group, which was similar to several series reported in the field of colorectal cancer $(13-16,19)$. And we found TD 

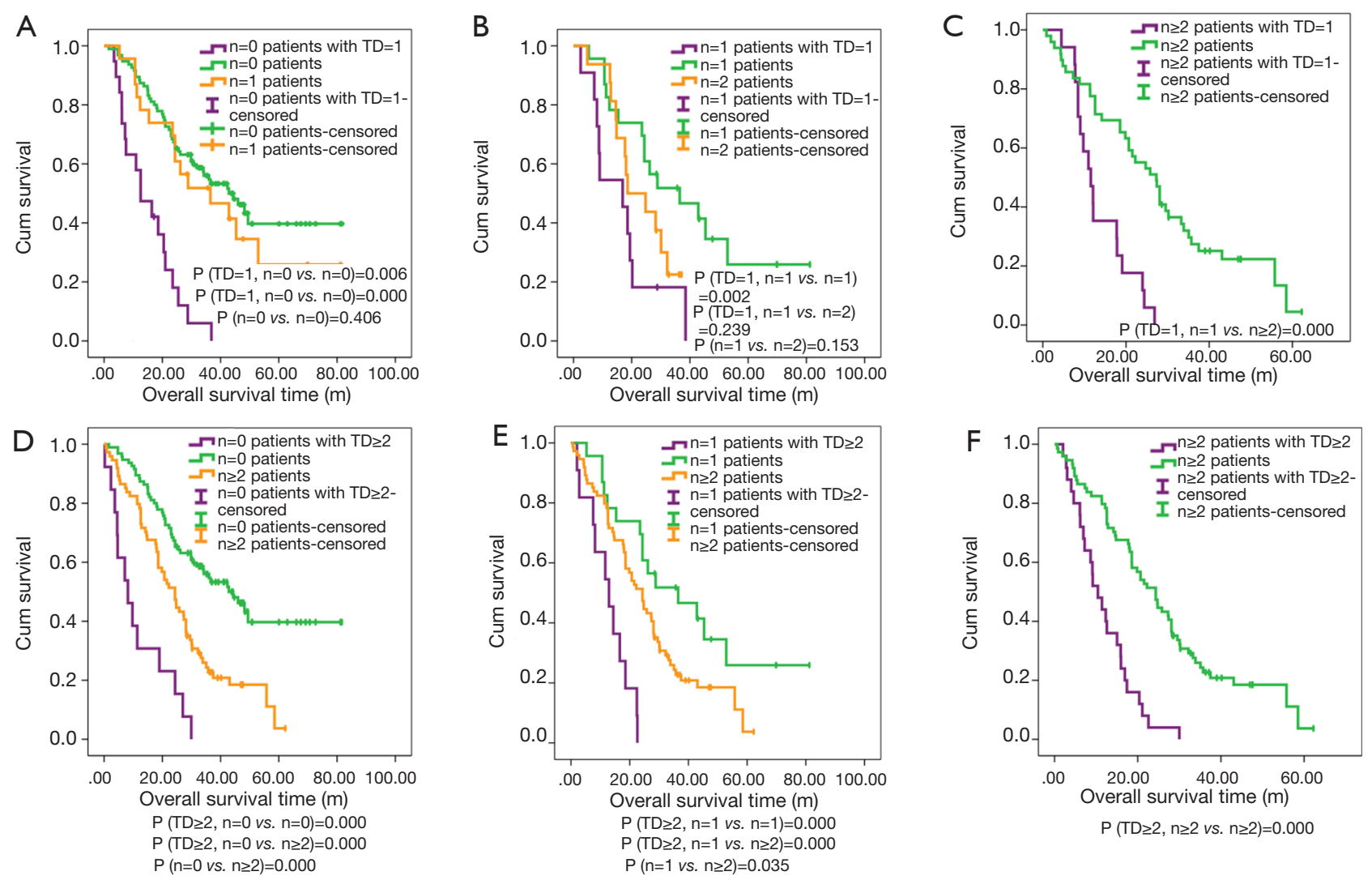

Figure 4 Comparison on prognosis of the number of tumor deposits and the number of metastatic lymph nodes. (A) Significant difference on prognosis between TD $=1, n=0$ group and $n=0$ group $(\mathrm{P}=0.006)$ as well as between $T D=1, n=0$ group and $n=1$ group were found $(\mathrm{P}=0.000)$; (B) no significant difference on prognosis was found between $\mathrm{TD}=1, \mathrm{n}=1$ group and $\mathrm{n}=2$ group $(\mathrm{P}=0.239)$ as well as between $\mathrm{n}=1$ group and $\mathrm{n}=2$ group $(\mathrm{P}=0.153)$ except for groups between $\mathrm{TD}=1, \mathrm{n}=1$ and $\mathrm{n}=2(\mathrm{P}=0.002)$; (C) significant difference on prognosis between $T D=1$, $\mathrm{n} \geq 2$ group and $\mathrm{n} \geq 2$ group was found $(\mathrm{P}=0.000)$; (D) patients in the group of $\mathrm{TD} \geq 2, \mathrm{n}=0$ got the significantly worst prognosis than patients in $\mathrm{n} \geq 2$ group than patients in $\mathrm{n}=0$ group (all $\mathrm{P}=0.000$ ); (E) patients in the group of $\mathrm{TD} \geq 2, \mathrm{n}=1$ got the significantly worst prognosis than patients in $\mathrm{n} \geq 2$ group than patients in $\mathrm{n}=1$ group; (F) significant difference on prognosis between $T D \geq 2, n \geq 2$ group and $n \geq 2$ group was found $(\mathrm{P}=0.000)$ (e.g., $\mathrm{TD}=1, \mathrm{n}=0$ : patients with one tumor deposit but without lymph node metastasis; $\mathrm{n}=0$ : $\mathrm{TDN}$ patients without metastatic lymph node; $n=1$ : TDN patients with one metastatic lymph node). TD, tumor deposit; TDN, tumor deposits negative.

could be considered as the independent prognostic factor in all patients with EC. Thus, the initial impression TD brings to us is that TD can be regarded as a good indicator, which distinguish the prognostic difference between TDP and TDN in patients with EC. However, how does TDP affect the prognosis and whether it can be seen as an apart of TNM stage system is the next point we focused on.

First the positive rate of TDs in patients with $\mathrm{EC}$ is $9.2 \%(96 / 1,044)$. The equally higher positive rate of TDs was seen in Nagtegaal (16) report who had integrated six studies with 1,520 patients of rectal cancer and concluded the positive rate of TDs in patients with rectal cancer was $26 \%(395 / 1,520)$. Chen (20) reported the positive rate of extranodal metastasis in gastric cancer was $9.1 \%$ $(133 / 1,457)$, which was almost the same as our research. Thus, the rare found in patients with EC can be elucidated by the lower rate of TD positive rate partially.

Second, as for the distribution of TDs in patients with EC, TDs were more found in lower part of esophagus $(12.9 \%)$ than in middle part (8.5\%)and the esophagogastric junction $(6.5 \%)$, but less in upper segment of esophagus (4.8\%) separately, however there're no significant difference among them. The results were as same as Sakai (7) study, who reported the positive lymph node capsular invasion was more found in lower part of esophagus (26.5\%) but less found in upper part $(23.1 \%)$, though there is no statistical 
difference neither. In rectum cancer, Prabhudesai (17) reported the mean number of TDs was found to be highest in tumors of the lower third of the rectum $(\mathrm{P}=0.002)$. At the same time, more tumors with middle $(10.5 \%)$ or whole part of gastric tube $(33.3 \%)$ location were significantly found with the extranodal metastasis in Chen (20) study ( $\mathrm{P}=0.001)$. Thus, it seems that the lower part of each digestive tract is more correlated with the occurrence of TDs.

When take $\mathrm{T}$ stage into consideration, we found the positive rate of TDs in $\mathrm{T} 3$ and $\mathrm{T} 4 \mathrm{a}$ stage were $11.54 \%$ and $11.44 \%$ respectively which were significantly higher than that in T2 stage (5.18\%) and T4b stage (1.14\%) $(\mathrm{P}=0.009)$. It can be concluded that deeper of the tumor infiltration, the more frequent TDs will be found. Equally, there're several series came to the similar results as our research reported, the tumor type is not the same though. Wang (21) retrospectively researched 1,829 patients with rectum cancer, and concluded the positive rate of TDs was higher in T3 stage (65.7\%) than in T2 stage $(17.3 \%)$ and T4 stage $(11.9 \%)$ but less in $\mathrm{T} 1$ stage $(5.2 \%)(\mathrm{P}<0.001 ; \mathrm{OR}=1.332$, 95\% CI: 1.123-1.579). In the meantime, more advanced $\mathrm{T}$ stage was also found in extranodal metastasis group in Chen study (20), which was also independently correlated to the extranodal metastasis of gastric cancer $(\mathrm{P}=0.001$; OR $=1.395,95 \%$ CI: $1.138-1.712$ ).

Forth, in our study, TDP rate in both metastatic and non-metastatic lymph node group was $13.9 \%$ and $5.4 \%$ respectively $(\mathrm{P}=0.000)$. Additionally, in our study, the TDs were connected with more advanced $\mathrm{N}$ stage significantly $(\mathrm{P}<0.001)$, meanwhile the same results were found in rectum cancer (14,22-25) and gastric cancer $(15,18,20,26,27)$, however, the $\mathrm{N}$ stage was not demonstrated to be related to TDs independently in our study but confirmed in Sakai (7) study $(\mathrm{P}=0.003$; HR $=1.089,95 \% \mathrm{CI}: 1.033-1.142)$, at the same time, Lee (26) study $(\mathrm{P}<0.001 ; \mathrm{HR}=5.024,95 \%$ CI 2.138-11.804) and Chen (20) study $(\mathrm{P}<0.001 ; \mathrm{OR}=1.357$, 95\% CI: 1.152-1.599) found N stage was related to TDs in gastric cancer independently as well.

Fifth, the poor prognosis of patients in TDP group can be observed via Kaplan-Meier curves and Cox proportional hazard regression model. And the prognostic significance of TDs was confirmed through the Cox proportional hazard regression $(\mathrm{P}=0.003$; HR $=2.633$, 95\% CI: 1.386-5.001), which was also demonstrated in few series about gastrointestinal surgery $(17,18,20,21)$. Furthermore, in order to investigate the relationship between TD and the TNM stage, the prognosis of each TNM stage had been shown in our study, in which the significant difference of prognosis was found in TNM II stage and TNM III stage with both $p$ value lower than 0.001 . Nevertheless, there's no significant difference of prognosis in TNM I stage and TNM IV stage $(\mathrm{P}=0.328)$. On the one hand, it is because that in our study, no TNM 0-I stage patients of EC with TDs were diagnosed pathologically, on the other hand, EC patients who had been diagnosed in TNM IV stage preoperatively were not suggested receiving the curative operation, thus the number of included patients in stage IV was little, additionally, the mortality rate of EC patients in TNM stage IV is higher than the patients in other stages according to the prognostic characters of EC (2), therefore the prognosis between TDP and TDN group in TNM stage IV was obscured. Then, the cross-stage comparison was performed and the results showed that patients of TDP group in TNM stage II harvested significant worse prognosis than patients of TDN group in TNM stage III $(\mathrm{P}<0.001)$, however there's no prognostic difference between patients of TDP group in TNM II stage and patients without TDs in TNM IV stage $(\mathrm{P}=0.886)$. At the same time, in order to clarify the TDP and lymph node metastasis, we firstly found that there's no significant difference on prognosis of TDP patients from $\mathrm{N} 0$ to N3 stage (Figure $3 A$ ) while the opposite result was found in TDN patients from N0 to N3 stage. Subsequently, the $1: 2$ propensity scoring matching was performed for comparing the prognosis between patients with TDs and lymph node metastasis purely, in which we concluded that the worst prognosis was always found in patients with at least 1 TDs whatever the number of metastatic lymph node is. And it can be regarded as a part of stage upgrading of EC. Sun (28) have once mentioned the stage migration of $\mathrm{TD}$ in gastric cancer, in which he reported the TDs as an independent prognostic factor played an important role on staging gastric cancer and he investigated all the cancers in T1-4a stage with TDs and reclassified them as T4a stage. Meanwhile, Lee (26) suggested the TDs should be included in the lymph node staging of gastric cancer because he found when add TDs into lymph node metastasis, the new formed $\mathrm{N}$ stage after stage migration was associated with patients' prognosis significantly. Thus, based on our study, TDs plays an important role on stage upgrading in EC, which we suggested TDs being regarded as an indicator of poor prognosis and stage migration.

Also, there're some limitations in our study. The origination of TDs in EC is still needed to research. In our study we have tried our best to retrospect the clinicopathologic features of EC patients with TDs in our hospital and analysis the results of their follow-up. At 
the same time, this study is from the single institute and the multicenter randomized controlled trial is required to confirm our results. Although the number of patients included in our study is more than 1,000 , the number of patients with TDs doesn't account for a large percentage of all patients, and in some subgroups the few patients might influence the final results.

\section{Conclusions}

In conclusion, the present results demonstrated the importance of TDs in patients with EC, which was confirmed an independent prognostic factor in esophageal cancer. Additionally, the patients in TDP subgroup had more advanced EC and worse prognosis than those in TDN subgroup. And it is more likely suggested TDs being regarded as an indicator of postoperative poor prognosis and stage migration in EC.

\section{Acknowledgements}

The authors thank Department of Pathology of West China Hospital, Sichuan University, China for the substantial work in diagnosing and examining the dissected tissues and lymph nodes pathologically.

Funding: This study was supported by the Natural Science Foundation of China-Guangdong Joint Fund (U1301227, U0932001), the National Science Foundation of China (81360331, 81472613, 81572341), and the Science \& Technology Planning Project of Guangdong Province (2014A030304060) and the Department of Education, Guangdong Government under the Top-tier University Development Scheme for Research and Control of Infectious Diseases.

\section{Footnote}

Conflicts of Interest: The authors have no conflicts of interest to declare.

Ethical Statement: The research was approved by the human participants committee of West China Hospital of Sichuan University (2005-126) and written informed consent was obtained from all patients.

\section{References}

1. Nakagawa S, Kanda T, Kosugi S, et al. Recurrence pattern of squamous cell carcinoma of the thoracic esophagus after extended radical esophagectomy with three-field lymphadenectomy. J Am Coll Surg 2004;198:205-11.

2. Daly JM, Fry WA, Little AG, et al. Esophageal cancer: results of an American College of Surgeons Patient Care Evaluation Study. J Am Coll Surg 2000;190:562-72; discussion 572-3.

3. Kato H, Fukuchi M, Miyazaki T, et al. Surgical treatment for esophageal cancer. Current issues. Dig Surg 2007;24:88-95.

4. Akiyama $\mathrm{H}$, Tsurumaru $\mathrm{M}$, Udagawa $\mathrm{H}$, et al. Radical lymph node dissection for cancer of the thoracic esophagus. Ann Surg 1994;220:364-72; discussion 372-3.

5. Isono K, Sato H, Nakayama K. Results of a nationwide study on the three-field lymph node dissection of esophageal cancer. Oncology 1991;48:411-20.

6. Wind J, Lagarde SM, Ten Kate FJ, et al. A systematic review on the significance of extracapsular lymph node involvement in gastrointestinal malignancies. Eur J Surg Oncol 2007;33:401-8.

7. Sakai M, Suzuki S, Sano A, et al. Significance of lymph node capsular invasion in esophageal squamous cell carcinoma. Ann Surg Oncol 2012;19:1911-7.

8. Amin MB, Edge S, Greene FL, et al. AJCC Cancer Staging Manual (M). 8th ed. New York: Springer, 2017:185-202.

9. Amin MB. The 2009 version of the cancer protocols of the college of american pathologists. Arch Pathol Lab Med 2010;134:326-30.

10. Fisher BJ, Perera FE, Cooke AL, et al. Extracapsular axillary node extension in patients receiving adjuvant systemic therapy: an indication for radiotherapy? Int J Radiat Oncol Biol Phys 1997;38:551-9.

11. Griebling TL, Ozkutlu D, See WA, et al. Prognostic implications of extracapsular extension of lymph node metastases in prostate cancer. Mod Pathol 1997;10:804-9.

12. Myers JN, Greenberg JS, Mo V, et al. Extracapsular spread. A significant predictor of treatment failure in patients with squamous cell carcinoma of the tongue. Cancer 2001;92:3030-6.

13. Gabriel WB, Dukes CE, Bussy HJ. Lymphatic spread in cancer of the rectum. Br J Surg 1935;23:395-413.

14. Goldstein NS, Turner JR. Pericolonic tumor deposits in patients with $\mathrm{T} 3 \mathrm{~N}+\mathrm{MO}$ colon adenocarcinomas: markers of reduced disease free survival and intra-abdominal metastases and their implications for TNM classification. Cancer 2000;88:2228-38.

15. Puppa G, Maisonneuve P, Sonzogni A, et al. Pathological assessment of pericolonic tumor deposits in advanced 
colonic carcinoma: relevance to prognosis and tumor staging. Mod Pathol 2007;20:843-55.

16. Nagtegaal ID, Quirke P. Quirke, Colorectal tumour deposits in the mesorectum and pericolon; a critical review. Histopathology 2007;51:141-9.

17. Prabhudesai A, Arif S, Finlayson CJ, et al. Impact of microscopic extranodal tumor deposits on the outcome of patients with rectal cancer. Dis Colon Rectum 2003;46:1531-7.

18. Puppa G, Ueno H, Kayahara M, et al. Tumor deposits are encountered in advanced colorectal cancer and other adenocarcinomas: an expanded classification with implications for colorectal cancer staging system including a unifying concept of in-transit metastases. Mod Pathol 2009;22:410-5.

19. Tateishi S, Arima S, Futami K, et al. A clinicopathological investigation of "tumor nodules" in colorectal cancer. Surg Today 2005;35:377-84.

20. Chen XL, Zhao LY, Xue L, et al. Prognostic significance and the role in TNM stage of extranodal metastasis within regional lymph nodes station in gastric carcinoma. Oncotarget 2016;7:67047-60.

21. Wang XN, Liang H, Wang JC, et al. Clinicopathological features and prognosis of 1829 patients with primary

Cite this article as: Shang QX, Yang YS, Xu LY, Li EM, Hu WP, Chen LQ. Prognostic significance and role in TNM stage of tumor deposits in esophageal cancer. J Thorac Dis 2017;9(11):4461-4476. doi: 10.21037/jtd.2017.10.60 colorectal cancer. Chin J Gastrointest Surg 2004;7:439-42.

22. Tocchi A, Mazzoni G, Lepre L, et al. Total mesorectal excision and low rectal anastomosis for the treatment of rectal cancer and prevention of pelvic recurrences. Arch Surg 2001;136:216-20.

23. Ratto C, Ricci R, Rossi C, et al. Mesorectal microfoci adversely affect the prognosis of patients with rectal cancer. Dis Colon Rectum 2002;45:733-42; discussion 742-3.

24. Ueno H, Mochizuki H. Clinical significance of extrabowel skipped cancer infiltration in rectal cancer. Surg Today 1997;27:617-22.

25. Reynolds JV, Joyce WP, Dolan J, et al. Pathological evidence in support of total mesorectal excision in the management of rectal cancer. Br J Surg 1996;83:1112-5.

26. Lee HS, Lee HE, Yang HK, et al. Perigastric tumor deposits in primary gastric cancer: implications for patient prognosis and staging. Ann Surg Oncol 2013;20:1604-13.

27. Jiang N, Deng JY, Ding XW, et al. Node-extranodal soft tissue stage based on extranodal metastasis is associated with poor prognosis of patients with gastric cancer. J Surg Res 2014;192:90-7.

28. Sun Z, Wang ZN, Xu YY, et al. Prognostic significance of tumor deposits in gastric cancer patients who underwent radical surgery. Surgery 2012;151:871-81. 